Ann. Biol. anim. Bioch. Biophys., I967, 7 (2), I63-172.

\title{
INFLUENCE DE L'AMIDON DE MAÏS ET DU SACCHAROSE SUR LA CONSOMMATION DES RÉGIMES SELON LE TAUX AZOTÉ: CONSÉQUENCES SUR LA COMPOSITION CORPORELLE DU RAT BLANC
}

\author{
B. DESMOULIN \\ avec la collaboration technique de M. Lecourtier et P. Peiniau \\ Station de Recherches sur l'Élevage des Porcs, \\ Centre national de Recherches zootechniques, 78 -Jouy-en-Josas \\ Institut national de la Recherche agronomique
}

\section{SOMMAIRE}

L'influence de la nature des glucides sur la consommation de nourriture, les performances de croissance et les caractéristiques de la composition corporelle a été étudiée chez le Rat blanc après le sevrage. A cet effet, l'amidon de maïs et le saccharose ont été introduits, seuls ou en association, dans des mélanges alimentaires renfermant I2, I4 ou i6 p. Ioo de protéines sous forme de caséine.

L'association des deux glucides en quantités égales dans les mélanges alimentaires permet d'augmenter le niveau spontané de leur consommation. A un taux azoté donné (I4 p. Ioo), la vitesse de croissance est améliorée de 22 p. 100 par rapport à celles observées lorsque les régimes contiennent un seul glucide, que celui-ci soit l'amidon ou le saccharose. D'autre part, l'évolution selon l'âge de la consommation après le sevrage provoque, avec les régimes riches en saccharose, une augmentation des teneurs en lipides des carcasses, lorsque le taux azoté est faible (I 2 p. I0o). Cette différence de la composition corporelle par rapport à celle obtenue avec les régimes riches en amidon n'apparaît pas lorsque le taux azoté est élevé (I6 p. I00).

\section{INTRODUCTION}

Les rats qui reçoivent après le sevrage un régime contenant comme seul glucide le saccharose s'y adaptent difficilement. D'après nos observations, leur consommation est hétérogène et en moyenne inférieure à celle des rats recevant un régime à base 
d'amidon et de saccharose. SpIVfy et al. (I958), WIENER et al. (I963) ont d'autre part indiqué une diminution de la consommation lorsque les régimes contiennent du saccharose seul, par rapport à ce qui se passe avec des régimes contenant soit de l'amidon de maîs, soit des dextrines. Toutefois, ScotT et al. (I947) n'observent pas chez le Rat de différences entre les quantités ingérées de régimes contenant soit le saccharose, soit 1'amidon de maìs. Ces différents effets sont attribués à l'appétence de 1'animal pour les différents glucides.

D'autre part, l'amidon (ou les dextrines) et le saccharose produisent un effet différent sur la croissance et la composition corporelle, en raison d'une action spécifique des glucides sur la consommation : à taux égal d'azote dans le régime, la vitesse de croissance est améliorée et la formation des dépôts lipidiques est plus importante avec les dextrines. HARPER et ELVEHJEM (I957) obtiennent des croissances identiques avec différents glucides en faisant varier le taux azoté des régimes. L'efficacité nutritionnelle des glucides est ainsi différente et elle varie selon le taux azoté. Toutefois, les observations effectuées par la méthode des bilans ne sont pas concordantes. Aux faibles taux azotés, SPIVEy et al. (I958) obtiennent une amélioration de la rétention azotée lorsque le saccharose est remplacé par l'amidon. Par ailleurs, WIENER et al. (I958) sur le Rat, SEWELL et MAXWELL (I966) sur le porcelet ont noté une diminution de la digestibilité apparente de l'azote avec les régimes à base de dextrines; ces auteurs n'obtiennent pas de différence entre les rétentions vraies d'azote, quelle que soit la nature des glucides.

L'objet de cette étude effectuée sur le Rat blanc après le sevrage est de préciser l'influence de l'amidon de maïs et du saccharose sur la consommation et le croît selon le taux azoté des régimes.

Les deux glucides ont été introduits seuls ou en association dans les régimes de façon à constituer à différents taux d'azote des mélanges glucidiques évoluant du saccharose pur à l'amidon pur. Les compositions corporelles obtenues avec les différents régimes sont comparées à poids final identique. Par ailleurs, les bilans d'azote et d'énergie sont calculés à partir des résultats de l'analyse corporelle.

\section{MATÉRIEL ET MÉTHODES}

\section{Animaux - Période pré-expérimentale}

70 rats mâles de souche Wistar, âgés de $22-23$ jours reçoivent durant une semaine un aliment granulé de sevrage en vue de connaître leurs potentialités de croissance. Au terme de cette période, les rats âgés de 4 semaines et d'un poids moyen de $66 \pm 5 \mathrm{~g}$ sont mis en lots d'après le poids et le gain de poids durant la période pré-expérimentale.

\section{Régimes - Schéma expérimental}

Sept régimes expérimentaux dont la composition est donnée dans le tableau I sont définis, pour un taux d'azote identique, par différentes proportions d'amidon et de saccharose : celles-ci évoluent de l'amidon pur au saccharose pur dans la composition des glucides totaux. La source azotée des régimes est constituée par la caséine additionnée de cystine $(2,5 \mathrm{p}$. 100 des protéines). 
Le schéma expérimental suivant indique la nomenclature des régimes selon leurs caractéristiques (taux azoté et composition glucidique):

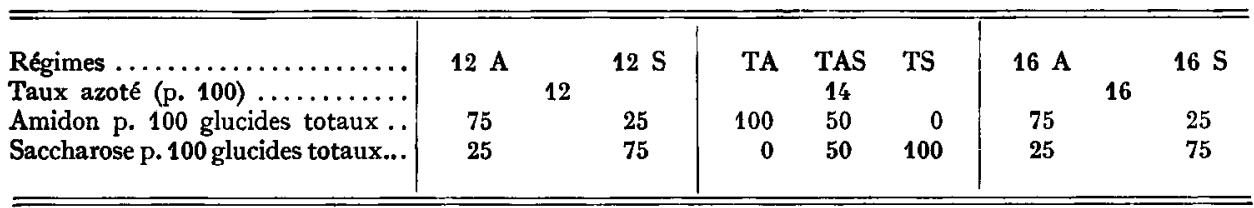

Les régimes TA, TAS, TS constituent, au même taux de I4 p. Ioo de matières azotées, une gamme témoin de l'influence des glucides seuls ou associés en quantités égales dans le mélange glucidique.

Les régimes à i 2 et $16 \mathrm{p}$. Ioo de protéines permettent d'étudier, selon deux taux azotés, l'influence des glucides associés en quantités inégales.

\section{Méthode d'étude - Interprétation}

Chacun des régimes est distribué à volonté aux animaux. Dans le lot ayant la vitesse de croissance la plus élevée, les animaux sont abattus après 5 semaines d'alimentation : le poids moyen atteint dans ce lot est choisi comme poids de référence pour les abattages effectués ultérieurement dans les autres lots.

L'influence de la composition des régimes sur la consommation et la croissance est étudiée au même âge (5 semaines), au même poids (référence d'abattage).

Les compositions corporelles sont comparées à poids final identique. Les rétentions d'azote et d'énergie sont calculées selon la méthode définie par Rérat et al. (1964). L'efficacité nutritionnelle est étudiée en calculant les coefficients de rétention correspondant aux coefficients d'utilisation pratique (CUP) de l'azote et de l'énergie selon TERroine et Valla (1933):

$$
\begin{gathered}
\text { CUP azote }=\frac{\mathrm{N} \text { retenu dans les tissus }(\mathrm{g}) \times 100}{\mathrm{~N} \text { ingéré }(\mathrm{g})} \\
\text { CUP énergie }=\frac{\text { Énergie retenue dans les tissus }(\mathrm{kcal}) \times \text { 100 }}{\text { Énergie ingérée }(\mathrm{kcal})}
\end{gathered}
$$

L'interprétation statistique des résultats est effectuée sur l'ensemble des régimes en comparant les moyennes (test de NeumanN-KeuLs). Par ailleurs, sur le schéma factoriel constitué par les régimes i $2 \mathrm{~A}$, I6 A, I2 $\mathrm{S}, \mathrm{I} 6 \mathrm{~S}$, l'influence de la composition du mélange glucidique est étudiée selon le taux azoté.

\section{RÉSULTATS}

\section{Composition des régimes. - Valeurs énergétique et azotée}

Le tableau I rapporte les résultats des analyses effectuées sur chaque régime : la teneur en matière sèche est obtenue à l'étuve (à Io3\%). La valeur énergétique brute, mesurée à la bombe calorimétrique, et la valeur azotée, obtenue par le dosage selon la méthode de $\mathrm{Kjeldahl,} \mathrm{ont} \mathrm{été} \mathrm{rapportées} \mathrm{à} \mathrm{la} \mathrm{matière} \mathrm{sèche} \mathrm{des} \mathrm{régimes.}$

Lorsque la proportion d'amidon augmente dans le mélange glucidique, la teneur en matière sèche des régimes diminue linéairement de $98 \mathrm{p}$. Ioo en présence de saccharose seul à 89 p. Ioo en présence d'amidon seul, inversement la teneur en énergétique 
brute de la matière sèche et sa teneur azotée augmentent lorsque l'amidon remplace le saccharose.

TABLEAU I

Composition des régimes - Teneur énergétique et azotée

\begin{tabular}{|c|c|c|c|c|c|c|c|}
\hline $\begin{array}{l}\text { Composition des } \\
\text { régimes }(\mathrm{g} / \mathrm{kg})\end{array}$ & $12 \mathrm{~A}$ & $12 \mathrm{~S}$ & $\mathrm{TA}$ & TAS & $\mathrm{TS}$ & $16 \mathrm{~A}$ & $16 \mathrm{~S}$ \\
\hline 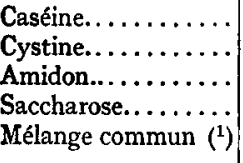 & $\begin{array}{r}137,5 \\
3,5 \\
531,8 \\
177,2 \\
150\end{array}$ & $\begin{array}{r}137,5 \\
3,5 \\
177,2 \\
531,8 \\
150\end{array}$ & $\begin{array}{r}160,6 \\
4,1 \\
685,3 \\
0 \\
150\end{array}$ & $\begin{array}{l}160,6 \\
4,1 \\
342,6 \\
342,7 \\
150\end{array}$ & $\begin{array}{l}160,6 \\
4,1 \\
0 \\
685,3 \\
150\end{array}$ & $\begin{array}{l}183,5 \\
4,7 \\
496 \\
165,8 \\
150\end{array}$ & $\begin{array}{r}183,5 \\
4,7 \\
165,8 \\
496,0 \\
150\end{array}$ \\
\hline $\begin{array}{l}\text { Teneur en M. S. }(\%) \\
\mathrm{N}(\mathrm{g}) / 100 \mathrm{~g} \text { M. S.. } \\
\mathrm{kcal} / 1000 \mathrm{~g} \text { M. S. }\end{array}$ & $\begin{array}{r}91,3 \\
2,01 \\
4708\end{array}$ & $\begin{array}{r}95,9 \\
1,95 \\
4549\end{array}$ & $\begin{array}{r}89,2 \\
2,39 \\
4802\end{array}$ & $\begin{array}{rl} & 93,7 \\
2,27 \\
4 & 686\end{array}$ & $\begin{array}{c}98,0 \\
2,19 \\
4485\end{array}$ & $\begin{array}{r}91,8 \\
2,75 \\
4716\end{array}$ & $\begin{array}{r}95,8 \\
2,59 \\
4575\end{array}$ \\
\hline
\end{tabular}

(1) Mélange contenant : huile arachide 80 , cellulose 20, mél. vitaminique 10, mél. minéral 40.

Consommations à âge égal (5 semaines)

Sur les graphiques de la fig. I, les consommations moyennes journalières de matière sèche sont représentées chaque semaine pour les différents régimes. D'après le graphique $I a$, au même taux azoté ( $\mathrm{r} 4 \mathrm{p}$. I0o), la consommation du régime qui contient une

(a)

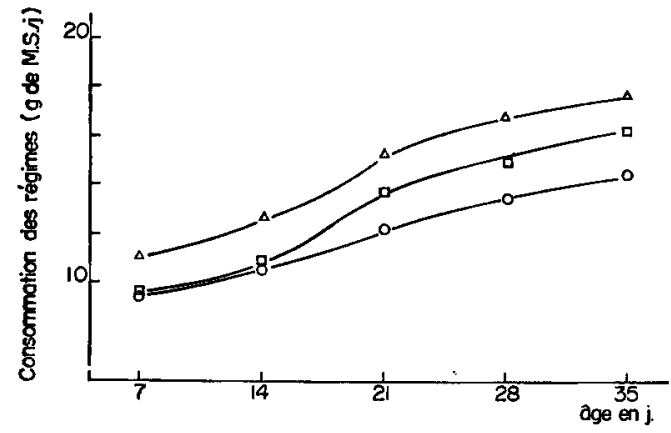

(b)

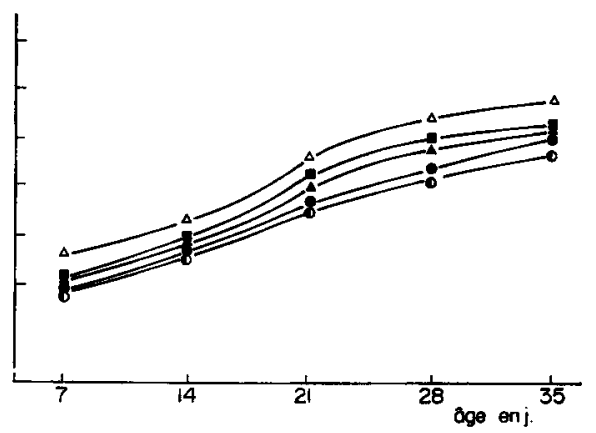

FiG. I. - Évolution selon l'âge de la consommation des régimes

$\begin{array}{ll}\text { A } & \text { TAS } \\ \text { T } & \text { TS } \\ \circ & \text { TA } \\ - & 16 \mathrm{~S} \\ - & 12 \mathrm{~S} \\ - & 16 \mathrm{~A} \\ - & 12 \mathrm{~A}\end{array}$

quantitéégale d'amidon et de saccharose est plus élevée que celles des régimes contenant l'amidon pur ou le saccharose pur ; entre ces deux derniers régimes, la consommation 
faible durant deux semaines devient ensuite significativement plus élevée seulement avec le régime contenant le saccharose pur. (graph. I $b$ ) : à deux taux azotés différents (I2 et I6 p. Ioo de protéines), les régimes riches en saccharose sont consommés en quantités plus importantes après deux semaines que les régimes riches en amidon.

Dans le tableau 2 sont rapportés les consommations journalières de matière sèche durant 5 semaines, ainsi que les ingérés d'azote et d'énergie correspondant à cette même période.

A taux azoté égal et en valeur relative, les consommations de matière sèche sont plus faibles de Io $\mathrm{p}$. IOo avec le saccharose seul et de $18 \mathrm{p}$. Ioo avec l'amidon

TABLEAU 2

Consommations journalières après 5 semaines et consommations totales à poids égal d'abattage

\begin{tabular}{|c|c|c|c|c|c|c|c|}
\hline Régimes & $12 \mathrm{~A}$ & $12 \mathrm{~S}$ & $\mathrm{TA}$ & TAS & TS & $16 \mathrm{~A}$ & $16 \mathrm{~S}$ \\
\hline $\begin{array}{l}\text { Consommations } \\
\text { journalieres } \\
d \grave{b} \text { semaines }\end{array}$ & & & & & & & \\
\hline $\begin{array}{l}\text { g M. S./jour ....... } \\
\text { mg N/jour........... } \\
\text { kcal/jour } \ldots . . . . .\end{array}$ & $\begin{array}{l}14,7^{*} \\
298 \\
69\end{array}$ & $\begin{array}{l}15,5^{*} \\
302 \\
70\end{array}$ & $\begin{array}{c}13,7 \\
328 \\
66\end{array}$ & $\begin{array}{r}16,8^{* *} \\
382 \\
79\end{array}$ & $\begin{array}{l}15,1^{*} \\
331 \\
68\end{array}$ & $\begin{array}{l}15,2^{*} \\
419^{*} \\
72\end{array}$ & $\begin{array}{c}16,0^{*} \\
414 \\
73\end{array}$ \\
\hline $\begin{array}{c}\text { Niveaux d'ingestion } \\
\text { de la M. S. (p. 100) } \\
\text { Consommations } \\
\text { totales d poids égal }\end{array}$ & 87 & 92 & 82 & 100 & 90 & 90 & 95 \\
\hline $\begin{array}{l}\text { Totale M. S. . ..... } \\
\text { Totale d'azote (g).. } \\
\text { Totale d'énergie kcal }\end{array}$ & $\begin{array}{c}620,4 \\
12,6^{*} \\
2921\end{array}$ & $\begin{array}{c}654,8 \\
12,8^{*} \\
2979\end{array}$ & $\begin{array}{r}637,5 \\
15,3 \\
3060\end{array}$ & $\begin{array}{r}588,8^{*} \\
13,4^{*} \\
2759 *\end{array}$ & $\begin{array}{l}701,1 \\
15,4 \\
3144\end{array}$ & $\begin{array}{c}570,6^{*} \\
15,7 \\
2691\end{array}$ & $\begin{array}{c}596,7^{*} \\
15,4 \\
2730^{*}\end{array}$ \\
\hline $\begin{array}{l}\text { Poids final }(g) \ldots . . . \\
\text { Age a a l'abattage }(\mathrm{j})\end{array}$ & $\begin{array}{r}274 \\
41\end{array}$ & $\begin{array}{r}270 \\
41\end{array}$ & $\begin{array}{r}271 \\
44\end{array}$ & $\begin{array}{r}273 \\
35\end{array} *$ & $\begin{array}{r}270 \\
44\end{array}$ & $\begin{array}{r}267 \\
37\end{array}$ & $\stackrel{270}{37^{*}}$ \\
\hline
\end{tabular}

Les moyennes marquées par un même astérisque $\left(^{*}\right)$ ne sont pas significativement différentes au seuil 0,05 .

seul que pour le régime contenant une quantité égale des deux sucres (TAS). A deux taux azotés différents, pour le mélange en quantités inégales des deux sucres, elles sont respectivement inférieures de 5 et 8 p. Ioo avec les régimes riches en saccharose, de ro et $\mathrm{I} 3 \mathrm{p}$. roo avec les régimes riches en amidon à celle du régime TAS.

A l'exception du régime TAS et malgré les différences de consommation de matière sèche notées précédemment, nous observons, à taux azoté égal, une égalisation des quantités d'énergie et d'azote ingérées. Lorsque le taux azoté varie, les variations de l'ingestion sont semblables avec les régimes riches en amidon ou en saccharose : 1 'influence de la composition du mélange glucidique sur les niveaux spontanés d'ingestion est ainsi indépendante de celle du taux azoté dans les limites considérées. 


\section{Consommation à poids égal}

Dans le tableau 2 sont rapportées les consommations totales de matière sèche, d'azote et d'énergie pour un même gain de poids. En particulier, selon 1'âge des animaux à l'abattage, on peut remarquer :

- Les consommations totales de matière sèche et d'énergie ainsi que les consommations journalières d'azote sont comparables lorsque 1'âge à l'abattage ne diffère pas significativement, ce qui est le cas pour les lots TAS, I6 A et I6 S d'une part, pour les lots I2 A, I2 S, TA et TS d'autre part. Entre ces lots, les consommations totales d'azote et les consommations journalières d'énergie sont toutefois très différentes.

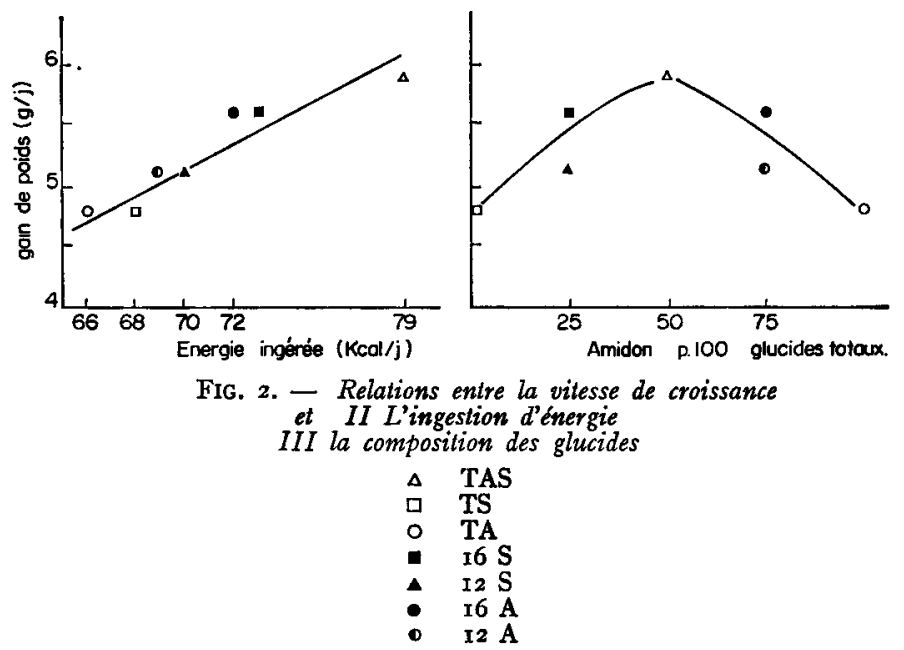

- pour un même taux de I4 p. Ioo de protéines, l'ingestion journalière d'énergie est le facteur déterminant de la croissance dans le lot TAS et le facteur limitant de la croissance dans les lots TA et TS. Ces résultats apparaissent sur le graphique (2) où sont mis en relation l'énergie ingérée et la vitesse de croissance dans les différents lots.

\section{Vitesse de croissance}

Les vitesses de croissance, après 5 semaines et à poids égal d'abattage, sont rapportées dans le tableau 3. Elles sont plus élevées dans les lots TAS, I6 A et I6 S que dans les autres lots.

TABLEAU 3

Vitesse de croissance $(\mathrm{g} / \mathrm{j})$

\begin{tabular}{c|c|c|c|c|c|c|c}
\hline Régimes & $12 \mathrm{~A}$ & $12 \mathrm{~S}$ & $\mathrm{TA}$ & $\mathrm{TAS}$ & $\mathrm{TS}$ & $16 \mathrm{~A}$ & $16 \mathrm{~S}$ \\
\hline & & & & & & & \\
\hline & & & & & & & \\
Après 5 semaines .. & 5,1 & 5,1 & 4,8 & $5,9^{*}$ & 4,8 & $5,6^{*}$ & $5,6^{*}$ \\
A poids final égal .. & 5,0 & 5,0 & 4,7 & $5,9^{*}$ & 4,6 & $5,4^{*}$ & $5,5^{*}$ \\
\hline
\end{tabular}


A poids égal, la vitesse de croissance des animaux du lot TAS est plus élévée de 22 p. roo que celle des animaux des lots TA et TS, abattus 9 jours plus tard. Nous avons porté sur le graphique (fig. 2) la vitesse de croissance en fonction de la teneur en amidon des mélanges glucidiques. On peut ainsi constater que la vitesse de croissance augmente lorsque les glucides sont associés dans le régime jusqu'à un maximum obtenu lorsque le mélange glucidique est constitué d'une quantité égale des deux sucres ; par ailleurs, les vitesses de croissance sont égalisées à taux azoté égal sauf pour le lot TAS.

\section{Composition corporelle. - Efficacité des rétentions}

Dans le tableau 4 sont présentés les résultats de l'analyse corporelle au poids final de $270 \mathrm{~g}$ atteint dans le lot TAS après 5 semaines d'expérience. Les teneurs en protéines et cendres ne diffèrent pas selon les régimes ; par contre, dans les lots TAS

TABLEAU 4

Compositions corporelles (p. 100 du poids frais) - Efficacité des rétentions

\begin{tabular}{|c|c|c|c|c|c|c|c|}
\hline Régimes & $12 \mathrm{~A}$ & $12 \mathrm{~S}$ & TA & TAS & TS & $16 \mathrm{~A}$ & $16 \mathrm{~S}$ \\
\hline P. 100 eau & 64,1 & $62,1^{*}$ & 64,0 & $61,3^{*}$ & 62,3 & 63,3 & 64,3 \\
\hline P. 100 cendres. .... & 3,0 & 3,0 & 3,1 & 2,9 & 3,1 & 2,9 & 3,0 \\
\hline P. 100 protéines $\ldots$ & 19,9 & 19,0 & 19,9 & 18,8 & 19,4 & 19,4 & 19,3 \\
\hline P. 100 lipides ..... & 13,0 & $15,9^{*}$ & 13,0 & $17,0^{*}$ & 14,3 & 14,4 & 13,4 \\
\hline CUP énergie.. & 18,1 & $19,5^{*}$ & 17,2 & $22,3^{*}$ & 17,3 & $20,5^{*}$ & $19,3^{*}$ \\
\hline CUP azote ... & $54,1^{* *}$ & $49,2^{*}$ & 44,1 & $47,0^{*}$ & 42,3 & 41,0 & 42,0 \\
\hline
\end{tabular}

Les moyennes marquées par un même $\left(^{*}\right)$ ne sont pas significativement différentes au seuil de 0,05 .

et I2 $\mathrm{S}$, les teneurs en lipides sont plus élevées et les teneurs en eau plus faibles que dans les autres lots et les différences sont significatives.

De plus, dans le schéma factoriel constitué par les lots à I2 et I6 p. roo de protéines, les régimes riches en saccharose provoquent, au faible taux azoté, une augmentation des teneurs en lipides des carcasses par rapport aux régimes riches en amidon. Cette influence n'apparaît pas avec les régimes à $I 6 \mathrm{p}$. Ioo de protéines et l'interaction est significative $(P=0,05)$.

Les valeurs des coefficients de rétention déduits des résultats de l'analyse corporelle ont été comparées globalement entre les différents lots : à durée d'entretien différente, les influences respectives de l'âge et du poids ne peuvent être dissociées. Cependant, à durée égale d'entretien, l'efficacité énergétique est plus élevée et l'efficacité azotée plus faible avec les régimes riches en saccharose (I2 $\mathrm{S}$ ) que pour les régimes riches en amidon (I2 A), lorsque le taux azoté est faible. Cette différence n'est pas observée aux taux azotés supérieurs à $12 \mathrm{p}$. Ioo de protéines. 
Remarquons enfin pour l'ensemble des régimes distribués à volonté que la rétention énergétique augmente avec l'ingestion journalière d'énergie. Par contre, la rétention azotée diminue lorsque l'ingestion journalière d'azote augmente.

\section{DISCUSSION}

\section{Cinétique de la consommation. - Adaptation aux glucides}

Après le sevrage et durant 5 semaines, un régime contenant une quantité égale d'amidon et de saccharose provoque une consommation de matière sèche plus élevée que les régimes dont la fraction glucidique est constituée par un seul sucre. Par ailleurs, après deux semaines, la consommation de matière sèche devient plus élevée avec le régime contenant le saccharose seul qu'avec celui contenant l'amidon de mais seul. Durant 4 semaines après le sevrage, REUSSNER et al. (I963) $n$ 'indiquent pas de différences de consommation entre des régimes à base d'amidon de mais ou de saccharose seul. Par contre, une stimulation de consommation est observée avec la dextrine de maïs. Les résultats de WIENER et al. (I963) coïncident avec ces observations pour la dextrine qui ne fait pas l'objet de notre étude. Ainsi les différences de niveaux d'ingestion attribuées aux glucides dépendent de leur nature d'une part, de la durée des périodes d'observation d'autre part.

L'évolution de la consommation avec l'âge, représentée sur les graphiques $I$, peut correspondre à une adaptation de l'animal aux quantités des sucres contenus dans son régime. Certains travaux concernant l'évolution selon l'âge des activités des systèmes enzymatiques peuvent justifier cette hypothèse : chez le Rat blanc après le sevrage, ISSELBACKER et SENIOR (I964) rapportent l'activité élevée de 1'amylase alors que celle de l'invertase se développe plus tardivement (BIAIR et al., Ig63). Par ailleurs, ces auteurs indiquent des possibilités d'induction d'activité des systèmes enzymatiques selon les autres composants des régimes. Cette observation peut être également applicable à l'association de l'amidon et du saccharose dans les mélanges glucidiques.

\section{Vitesse de croissance. - Efficacité alimentaire}

Pour l'ensemble des régimes, la vitesse de croissance est en relation directe avec les niveaux de consommation et en particulier avec les consommations journalières d'énergie : ainsi, pour un même apport des principes énergétiques relativement aux principes azotés de 1'aliment, dans les lots à I4 p. Ioo de protéines, la vitesse de croissance est plus élevée de $22 \mathrm{p}$. Ioo avec les régimes contenant les deux sucres que pour les régimes contenant 1'amidon ou le saccharose pur. D'autre part, la vitesse de croissance n'est pas en relation directe avec les consommations journalières d'azote : à un taux supérieur à I2 $\mathrm{p}$. Ioo de protéines, la rétention azotée diminue et il apparaît une utilisation des protéines à des fins énergétiques (BOSSHARDT et BARNES, I946).

A durée égale d'entretien, l'efficacité azotée est plus élevée avec les régimes riches en amidon, aux faibles taux azotés seulement. Ce résultat est en concordance avec les données de Marshal L, et Womack (r954) et de Spivey (I958) qui ont observé, 
avec l'amidon, une épargne de la dépense azotée lorsque le taux azoté est faible. Toutefois, cela n'est pas confirmé par WIENER et al. (I963). Plusieurs mécanismes peuvent être invoqués pour expliquer les différents effets des glucides sur l'efficacité azotée. Des effets spécifiques ont été signalés sur la vitesse du transit gastro-intestinal (DREISSBACK-NASSET, I954) ou sur les mécanismes de la digestion et de 1'absorption (DAHLQVIST et al., I963). D'autre part, le milieu intestinal et notamment la microflore sont modifiés (WISEMAN, I953) : ainsi, 1'amidon favoriserait plus que le saccharose les synthèses microbiennes par la microflore. Cette activité est bénéfique pour l'animal, notamment en ce qui concerne les synthèses vitaminiques (RÉRAT, I964). Enfin, HaRper et Katayama (I953), Chang (I965) démontrent chez le Rat que la méthionine du régime est épargnée en présence d'amidon de mais. Ces différents mécanismes peuvent ainsi entraîner la supériorité de l'efficacité nutritionnelle des régimes riches en amidon sur les régimes riches en saccharose lorsque le taux azoté est faible.

\section{Composition corporelle. - Taux azoté des régimes}

La composition corporelle résulte des influences du niveau de la consommation et de ses variations dans le temps.

- Lorsque le niveau est très élevé durant toute la croissance, on observe une augmentation des teneurs en lipides des carcasses pour les animaux qui reçoivent le régime contenant une quantité égale de deux glucides; l'adiposité des animaux est due à une ingestion élevée d'énergie et simultanément le coefficient de rétention énergétique est élevé.

- Lorsque le niveau de la consommation varie au cours de la croissance, il est intéressant de se référer à l'influence de la nature des glucides sur l'évolution de la consommation selon 1'âge : le saccharose provoque une évolution du type Bas-Haut, alors que l'amidon entraîne comparativement une courbe de consommation du type Haut-Haut. Avec un régime contenant $x 6$ p. Ioo de protéines, le niveau de l'ingéré azoté reste au-dessus des besoins de croissance, alors qu'il est en dessous de ces besoins avec un régime contenant $\mathrm{I} 2 \mathrm{p}$. Ioo de protéines, en particulier avec le régime riche en saccharose au début de la croissance. Avec ce régime, la courbe de consommation provoque de plus en fin de croissance une augmentation des niveaux énergétiques. L'adiposité des animaux est alors accrue. Conformément à nos études sur les variations de la consommation d'énergie par un rationnement du type Bas-Haut (non publié), le coefficient de rétention énergétique est amélioré.

Selon 1'interprétation de HARPER (Ig63), l'importance des dépôts lipidiques à poids égal d'abattage doit être attribuée à une ingestion élevée d'énergie par rapport au poids de l'animal. Nos observations indiquent de plus qu'une augmentation des dépôts lipidiques peut résulter d'un déséquilibre entre le niveau des ingérés énergétiques et le niveau des ingérés azotés selon l'âge.

Reçu pour publication en avril 1967. 


\section{SUMMARY}

\section{INFLUENCE OF MAIZE STARCH AND SACCHAROSE ON INTAKE OF FOOD BY THE WHITE RAT} AFTER WEANING : EFFECT ON BODY COMPOSITION

After weaning white rats of the Wistar strain were fed to appetite on synthetic diets with 12 or 16 per cent protein supplied by casein. Carbohydrate in the diets was maize starch and saccharose in proportions $75: 25$ or $25: 75$. Control diets, with 14 per cent protein, had only starch or saccharose or equal proportions of each.

On diets with 14 per cent protein equal proportions of the two carbohydrates gave greater intake and growth rate was 22 per cent more than when the diet had only one carbohydrate, either starch or saccharose. When animals were killed at the same weight carcasses had greater lipid content when growth rate was high and this could be attributed to excessive intake of energy in relation to bodyweight of the animal.

With 12 or I 6 per cent protein combination of the two carbohydrates in unequal proportions in the feed gave different intakes depending in the nature of the carbohydrate. After two weeks intake of dry matter increased more quickly on diets with the greater proportion of saccharose than those with more starch. Thus the intake curve was of the type Low-High for the first diet and HighHigh for the second. At the same slaughter weight there was an increase in lipid content of carcases of rats given 12 per cent protein when the diet had more saccharose; there was no such difference dependent on the type of carbohydrate when the diet had 16 per cent protein. Observations of the form of the intake curve show that the increase of lipid deposited could be the result of imbalance of intakes of energy and protein according to age.

\section{RÉFÉRENCES BIBLIOGRAPHIQUES}

Blair D. G. R., Yakihets W., TUBA J., I963. Rat intestinal sucrase : the effects of rat age and sexe and of diet on sucrase activity. Canad. J. Biochem., 41, 91 7-929

BosshardT D. K., Winifred P., O'Doherty Katleen, Barnes R. H., I946. Influence of calorie intake on the growth utilization of dietary protein. $J . N u t r ., 32,641-65 \mathrm{I}$.

Chang Y. O., I 965 . Excretion of total nitrogen, lysine and methionine by rats as affected by the type of carbohydrate in the diet. J. Nutr., 85, $207-212$.

Darrqvist A., Thomson D. L., r963. The digestion and absorption of sucrase by the intact rat. $J$. Physiol., 167, 197-209.

Dreissbach L., NASset E. S., 1954. Absorption of carbo Hydrate and protein as offected dy feeding cornstarch, banana or glucose $f$. Nutr., 58, 523-532.

Harper A. E., ElvehJEm C. A., I957. A review of the effects of differents carbohydrates on vitamin and amino-acid requirements. J. Agric. Food. Chem., 5, 754-758.

Harper A. E., Katayama M. C., I953. The influence of various carbohydrates on the utilization of low protein rations by the white rat. I. Comparison of sucrase and cornstarch in 9 p. roo casein ration. $J$. Nutr., 49, 26I-275.

KURT J., ISSELBACKER R., JOAHN-SENIOR M. D., I964. The intestinal absorption of carbohydrate and fat. Gastroenterology, 46, 287-298.

MaRshald M. W., WoMACK M., I954. Influence of carbohydrate, nitrogen source and prior state of nutrition on nitrogen balance and liver composition in the adult rat. J. Nut., 52, 51-73.

RERAT A., 1964. Rôle de la flore digestive dans la production des vitamines chez les monogastriques et les polygastriques. Journées Scientifiques : récents progrès en vitaminologie. Ann. Nutr. Alim., 18, 187-263.

Rérat A., FÉrier C., Henry Y., Lougnon J., 1964. Évolution de la composition corporelle chez le rat blanc en croissance. Ann. Biol. anim. Bioch. Biophys., 4, 35-47.

Reussner J. R., AnDros J., Thiessen R., 1963. Studies on the utilization of various starches and sugars in the rat. J. Nutr., 80, 281-298.

Scott E. M., Ethel L., Verney., I947. Appetite for carbohydrates. J. Nutr., 34, 401-407.

SEwell R. F., Maxwell C. V., 1966. Effects of various sources of carbohydrates in the diet of earlyweaned pigs. J. anim. Sci., 25, 796-799.

Spivey H. E., Katayama M. C., Yoshida M., HARPER A. E., I958. Significance of the a protein-sparing effect of dextrin. Am. J.Physiol., r93:479-482.

Terroine E. F., Valla S., 1933. La valeur comparée des différents aliments protéiques dans la croissance. C. R. Acad. Sci., 196, 288-290.

Wiener Ruta P., Yoshida M., HaRPer A. E., 1963. Influence of various carbohydrates on the utilization of low protein rations by the white rat. J. Nutr., 80, 279-289.

Wiseman R. F., r953. Study on the intestinal microflore of chickens. Thesis Univ. Hawai. 